\title{
B mode, Doppler and ultrasound elastography imaging on active trigger point in women with myofascial pain syndrome treated by dry needling
}

\author{
H. Adigozali ${ }^{1}$, A. Shadmehr², E. Ebrahimi ${ }^{3}$, A. Rezasoltani ${ }^{4}$, F. Naderi ${ }^{5}$ \\ 1 Physical Therapy Department, School of Rehabilitation, Tabriz University of Medical Sciences, Tabriz, Iran \\ 2 Physical Therapy Department, School of Rehabilitation, Tehran University of Medical Sciences, Tehran, Iran \\ 3 School of Rehabilitation, Iran University of Medical Sciences, Tehran, Iran. \\ ${ }^{4}$ Physiotherapy Department, School of Rehabilitation, Shahid Beheshti University of Medical Sciences, Tehran, Iran \\ 5 Radiology Department, Iran University of Medical Sciences, Tehran, Iran
}

\section{CORRESPONDING AUTHOR:}

Azadeh Shadmehr

Vice Dean for research \&

International affairs

of School of Rehabilitation,

Tehran University of Medical

Sciences, Enghelab Ave, Pich

Shemiran, Tehran

Phone: (9821)77528468.

E-mail: shadmehr@tums.ac.ir

DOI:

10.32098/mltj.03.2019.16

LEVEL OF EVIDENCE: 3

\begin{abstract}
SUMMARY
Introduction. Myofascial pain syndrome is a common regional pain characterized with myofascial trigger points (MTrP) in the skeletal muscle fibers. Deep dry needling (DDN) is an effective method used by variety of clinicians in deactivation of these points. The aim of present study was to determine clinical effectiveness and to evaluate gray scale ultrasonography, Sono Doppler and ultrasound elastography images of upper trapezius following the DDN.

Methods. Thirteen women with MTrP in the upper trapezius $(28.85 \pm 7.70$ years old) participated in the present study. Participants were treated with DDN during one session. Clinical outcomes were cervical range of motion (ROM), pain intensity, and pain pressure threshold (PPT). Ultrasonographic outcomes were MTrP area, intramuscular blood circulation and upper trapezius thickness and stiffness.

Results. A significant decrease of pain intensity was observed after intervention in all subjects $(\mathrm{p}<.001)$. PPT and side bending cervical ROM had significant improvement in post treatment $(\mathrm{p}<.001)$. Subjects showed significantly lower stiffness and higher intramuscular circulation immediately after the DDN $(\mathrm{p}<.001)$.

Conclusions. The present study demonstrated that DDN had immediate influences on muscle circulation, viscoelastic properties of the upper trapezius, and clinical outcome measurements. The findings of this study provide objective evidence of the effectiveness of DDN.
\end{abstract}

\section{KEY WORDS}

Myofascial pain syndrome; dry needling; ultrasonography; blood flow; muscle stiffness

\section{BACKGROUND}

Myofascial pain syndrome (MPS) is one of the most common complaints in the field of musculoskeletal dysfunction (1). This syndrome is associated with the presence of myofascial trigger points (MTrPs) with a prevalence as high as $95 \%$ (2-4). MTrPs are palpable tender spots that are localized in a taut band of skeletal muscle (5). Consequences of the formation of MTrPs in affected muscles include reduced fascia elasticity, restricted range of motion (ROM), increased muscle tension, local and referral pain, as well as abnormal autonomic phenomena (2). The upper trapezius is one of the most common muscles associated with this syndrome.
Based on the clinical findings, there are two distinct forms of MTrPs, active and latent. In symptomatic subjects with an active MTrPs, pain is reproduced spontaneously or with movement and by applying compression, but in the latent form, spontaneous pain is not observed (7). It has been suggested that some conditions, such as direct trauma or sustained muscle contractions without adequate recovery time, may develop intramuscular pressure high enough to diminish the muscle blood circulation potentially encouraging the formation of trigger points $(1,8)$. According to previous studies, affected muscle with MPS has different blood flow in comparison with healthy muscle, then moni- 
toring the blood flow characteristics can be useful to investigate overtime changes or after therapeutic intervention 4 . Also, the chemical environment of active MTrP was found to be different from the normal surrounding tissue $(9,10)$. These chemical changes could stimulate nociceptors as the source of continuous pain of active MTrPs and may lead to ischemic condition around them (11). Persisting pain from these MTrPs may lead to abnormal adaptation in the dorsal horn of the spinal cord, thus treatment becomes more difficult $(7,9)$. Moreover, the active MTrP may be associated with changes in the viscoelastic properties of the muscle fibers. These changes are characterized by hard taut band of muscle fiber, which alter muscle stiffness and reduce the $\operatorname{ROM}(7,12)$. Because, various studies have shown an increase in muscle stiffness and decrease in blood flow in the MPS, implementation of treatment strategies to counteract these changes are necessary $(12,13)$.

Many treatment methods have been developed to eliminate MPS symptoms. Dry needling is one of the most commonly used techniques in MPS treatment (14). In deep dry needling (DDN), in order to elicit a local twitch response (LTR), the needle is inserted into the MTrP. This response is associated with reduction of muscle stiffness which is palpable and pain relief (12). Also, some studies have shown that DDN can improve intramuscular blood circulation $(9,13)$. Although the actual working mechanism of DDN is still being debated, there are comparative studies regarding the effects of dry needling versus other treatments of MPS (12-15). Cumming et al.'s systematic review demonstrated that DDN is an effective treatment approach for $\mathrm{MTrP}$ (16). Further, Gerber et al. demonstrated sustained reduction of pain intensity after treatment by DDN at 6 weeks follow up (17).

Despite the clinical efficacy of various treatments for alleviating the symptoms of $\mathrm{MTrP}$, objective quantification of the severity of MPS in terms of blood flow and muscle stiffness is necessary. Diagnostic ultrasonography is suitable for noninvasive real-time imaging of muscle, fascia, tendon and blood vessels. Also, ultrasound elastography is an imaging technique which is newly used for scanning muscles, and quantifying muscle stiffness $(16,18-20)$. Ultrasonography may be helpful in overcoming the subjective weaknesses of diagnosing MPS (especially in deep muscles in which palpation is challenging) and effectively monitoring the clinical outcomes of different treatments $(7,18)$. Only few researches thus far have evaluated muscle stiffness and blood flow in the MPS using ultrasonography $(2,4,12)$.

Although DDN is considered to be an effective method for MPS treatment, to the authors' knowledge, there is no comprehensive assessment regarding the clinical (subjective) and ultrasonographic (objective) features of this meth- od. Therefore, we believe that this study is the first research designed for the purpose of evaluating both clinical effects (e.g., cervical ROM, pain intensity, and pain pressure threshold (PPT) and ultrasonographic changes (e.g., morphometric, muscle stiffness, and muscle blood flow) after DDN treatment in female patients with MPS. The objective of this study was to evaluate the effects of DDN on clinical outcomes and muscle thickness, area of MTrP, blood flow, muscle stiffness in women presenting MPS in the upper trapezius.

\section{METHODS AND MATERIALS}

\section{Subjects}

Thirteen right-handed females with MPS, between 20 and 44 years of age, and within the normal range of body mass index participated in the present study. Patients referred to physical therapy clinics due to neck or shoulder pain were invited by announcements in clinics of the rehabilitation school. Subjects were eligible for current study if they had an active $\mathrm{MTrP}$ in right upper trapezius muscle. Patients diagnosed with fibromyalgia syndrome, trigger point injection in the upper trapezius, cancer, history of surgery, direct trauma and fracture in the shoulder or cervical spine, radiculopathy, whiplash injury, degenerative diseases in the cervical vertebrae, pregnancy, local infection and taking anticoagulants were excluded. After an initial assessment, the objective of the study was explained to the subjects. They signed a consent form and were entered into the study. This study was approved by the ethics committee of the Tehran University of Medical Sciences University of Medical Sciences. Current study meets the ethical standards of Muscle, Ligaments and Tendons Journal (21). Clinical and ultrasonographic assessments were performed in one session (these tests were conducted before and after treatment). Patients were treated by one session of DDN.

\section{CLINICAL ASSESSMENT}

The presence of active MTrP in patients was confirmed by clinical evaluation by an expert physical therapist. The following criteria were used in the volunteers: a history of cervical pain for at least 12 weeks, the existence of a palpable taut band in the upper trapezius, and the presence of only one palpable hypersensitive spot in the taut band (halfway between the acromion process of the right scapula and $\mathrm{C} 7$ vertebra). To separate active from latent MTrP, a familiar referral pain must have been reproduced by applying pressure to the tender spot. This test was done with an algometer at an approximate pressure of $2.5 \mathrm{~kg} / \mathrm{cm}^{2}$ to the MTrP (22). The midpoint 
of the upper trapezius was identified in all the participants as follows: bony landmarks, including the spinous process of the 7 th cervical vertebra $(\mathrm{C} 7)$ and the acromion process of the scapula in the right shoulder were marked (figure 1). Thereafter, the distance between these two points was measured using a measuring tape and their midpoint was determined on the skin with a maker. This point was used for similar assessments at baseline and post-treatment examinations. The presence of $\mathrm{MTrP}$ in participants was confirmed by manual palpation at this point, after which, clinical outcome measurements, such as active bilateral cervical side bending, PPT, and pain intensity were examined.

Assessment of cervical ROM. The range of active cervical movements, including contralateral and ipsilateral side bending were measured using a dual inclinometer ( $\mathrm{J}$-Tech medical, US) with the subject sitting upright on the chair. These tests were done on all subjects with three repetitions and the average value was used for analysis.

Assessment of PPT. In order to evaluate the PPT, the digital algometer (J-Tech medical, commander power track, US) was used. The circle disk with a width of $1 \mathrm{~cm}$ was placed perpendicular to the specified point, and pressure was increased slowly until pain was felt and subjects indicated by saying "yes". The average value of three measurements was reported as PPT and the interval between repetitions was $30 \mathrm{~s}$ (23). Assessment of pain intensity. To provoke local and referral pain of MTrP, a digital algometer was used and a pressure of $2.5 \mathrm{~kg} / \mathrm{cm}^{2}$ was applied to the marked spot. Thereafter, subjects were instructed to select the level of pain by marking along the visual analogue scale (VAS). VAS is a horizontal line grading from 0 to 10 ( 0 , the minimum level of pain; 10 , the maximum level of pain).

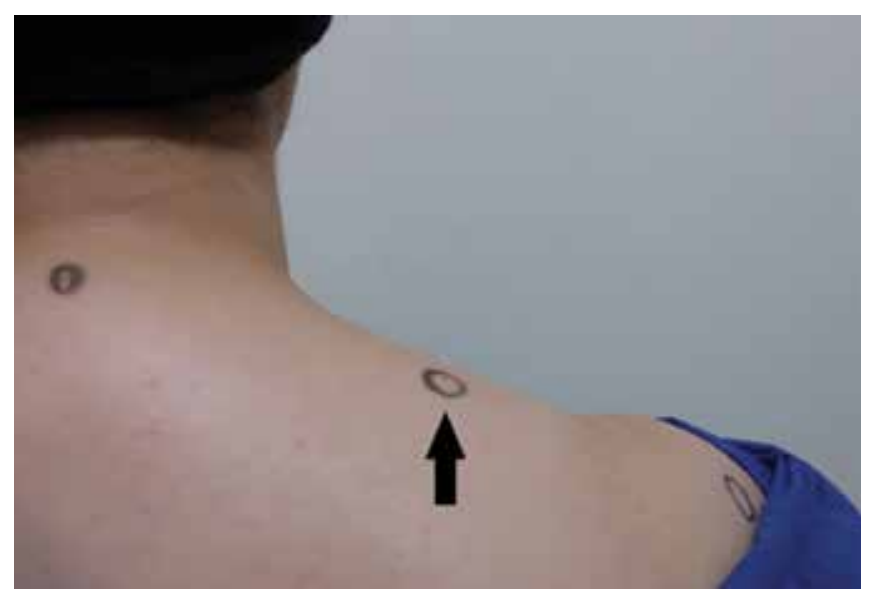

Figure 1. The midpoint of the spinous process of the 7th cervical vertebra (C7) and the acromion process of the scapula in the right shoulder were marked in all participants.

\section{ULTRASONOGRAPHY SCANNING}

Ultrasonographic features of the upper trapezius, including B-mode imaging, ultrasound elastography and Doppler indices were measured. Ultrasonography was performed after 30 minutes of clinical evaluation. Ultrasonography assessments were done with the following steps: 1) gray- scale; 2 ) ultrasound elastography; 3 ) Color Doppler imaging.

Each subject underwent an ultrasonic evaluation using a clinical ultrasonic device (My lab70, xvision, Italy) with a linear probe $(7-11 \mathrm{MHz})$ targeted at the marked point. All images were captured by an experienced sonographer in the field of musculoskeletal imaging. In the B-Mode gray scale, the ultrasound probe was placed parallel to the muscle fibers of the upper trapezius. The vertical distance between echogenic fascial layers was measured as muscle thickness. The upper trapezius muscle thickness was measured at rest position. Participants were instructed to sit and be relaxed with $0^{\circ}$ of shoulder abduction while their forearms were on the armrest of the chair and in the pronation position (figure 2). In gray scale, the MTrP appeared as a dark nodule in the muscle image. The area of this nodule in the upper trapezius muscle was determined in the participants (figure 3).

Muscle stiffness (tissue deformation in response to compression force) was measured with an ultrasound elastogram at the same position as the previous test in resting position. The ultrasound transducer was located perpendicular to the upper trapezius muscle in the marked region and was compressed rhythmically (with a frequency of $2 \mathrm{~Hz}$ ) by a sonographer $(2,23)$. A pressure indicator item in the ultrasonogram was used for controlling the intensity of manual compression force. Color -coded images were superimposed on gray scale images during the compression. In this

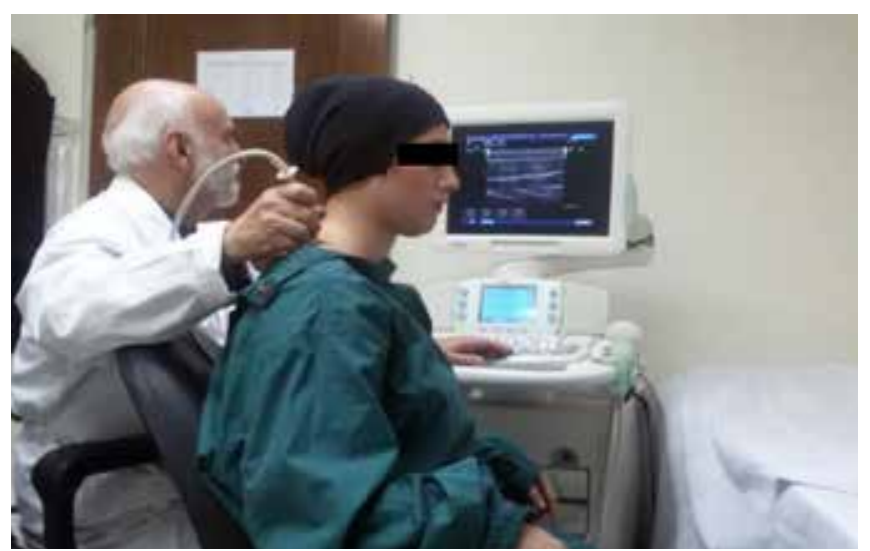

Figure 2. The position of patient is shown for clinical evaluation and ultrasound imaging. Participants were instructed to sit while their forearms were on the armrest of the chair and in the pronation position. 


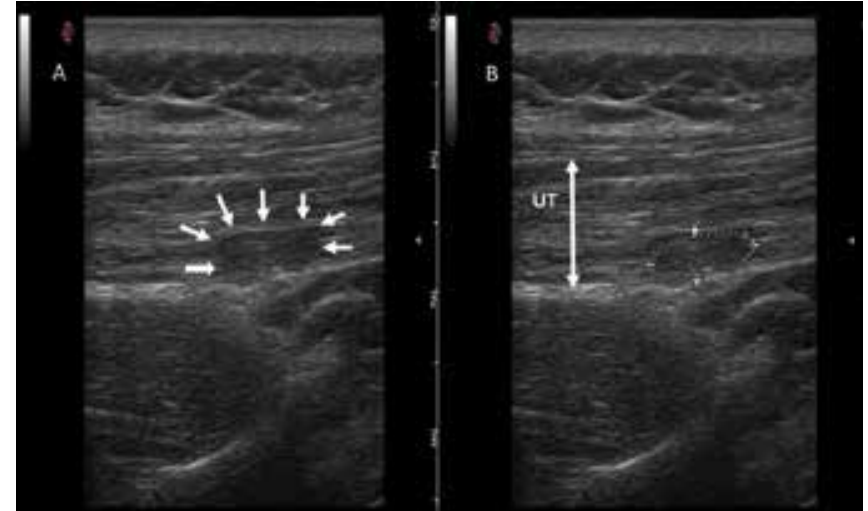

Figure 3. gray scale ultrasound images from the patient participant. Area of myofascial trigger point was calculated. UT: upper trapezius

design, softer parts were coded as green while stiffer parts were coded as red. With the selection of proper image, two circle regions of interests (ROI) with a size of $5 \mathrm{~mm}^{2}$ were adjusted on the frozen image. Reference ROI was adjusted in the subcutaneous space and another ROI was placed in the upper trapezius muscle in the vicinity of the trigger point zone. Muscle stiffness was calculated by dividing the strain ratio of the muscle zone by the reference zone in the ultrasound software (see figure 4). Increased strain ratio implied decreased muscle stiffness (23).

The upper trapezius circulation was scanned with color Doppler ultrasonography. The spectral Doppler waveforms

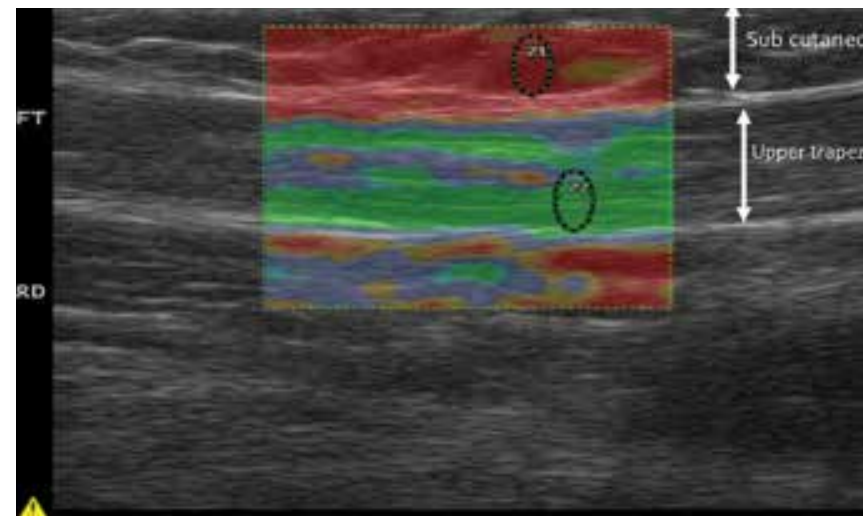

Figure 4. Ultrasound elastography of upper trapezius. A region of interest (ROI) was placed in the (Z1) sub cutaneous space and another ROI was adjusted in the (Z2) bulk of upper trapezius, then the strain ratio was calculated as Z2/Z1. The ultrasonogram image is superimposed onto the gray scale image, displays a color-coded map of elasticity. The color bar in the left of the screen presents the stiffness of the tissues within the ROI, ranging from green (soft) to red (hard). were used to determine the maximum velocity (PSV), minimum velocity (EDV) and RI of each cardiac cycle. The RI is defined as a ratio that is obtained by the difference between peak systolic and end diastolic velocities to the peak systolic velocity. For this study setup, these parameters were calculated in the arterioles or arteries found in the MTrP vicinity of the upper trapezius in the images (figure 5).

The reliability of ultrasonographic scanning of the upper trapezius including muscle thickness, area of MTrP, muscle stiffness and blood flow (Peak Systolic Velocity (PSV), End Diastolic Velocity (EDV) and Resistive Index (RI)) were examined in healthy subjects and patients with MPS in our previous studies $(24,25)$. We previously demonstrated that all variables, except EDV, had an excellent reliability $(>0.806)$ for the patient group. The Intra-class Correlation Coefficient (ICC) for EDV was 0.738 , and was considered as a poor to good reliability. For healthy subjects, the value of ICC for total variables showed an excellent level of reliability. EDV had the lowest reliability (ICC=0.771) among them.

\section{INTERVENTION}

The participants were treated with DDN during one session. The patients were instructed to lie with a neutral head position. They were treated with DDN using sterilized stainless steel needles $(50 \times 0.25 \mathrm{~mm}$ (Dong - Bang, Korea)). The $0.25 \mathrm{~mm}$ size minimized the chances of needle induced pain. At first, a marked point on the skin was sterilized and then this site was pinched between the left index finger and thumb of the physical therapist. The needle was inserted vertically into the skin to get into the trigger point (the correct position was confirmed by reproduction of familiar referral pain or visible/palpable LTR). The needle was manipulated rapidly forward and backward until no more

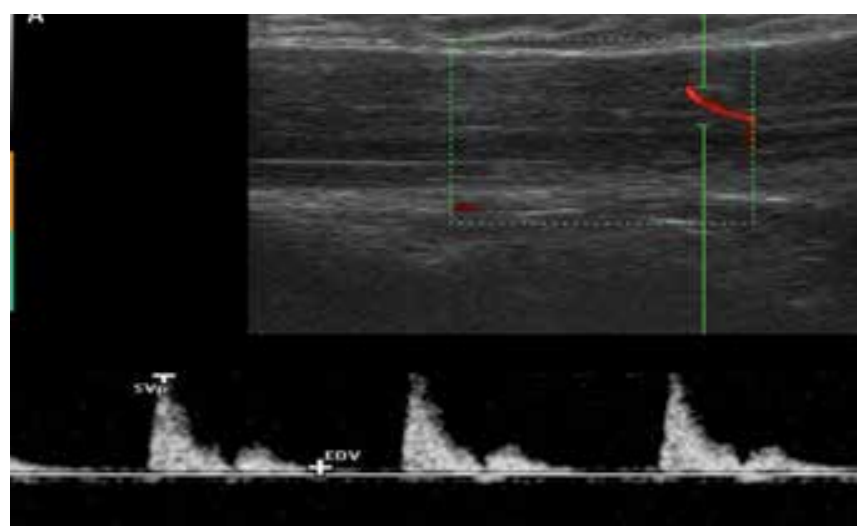

Figure 5. Sono Doppler imaging of blood flow through the upper trapezius muscle. PSV and EDV are shown in the blood flow waveform. 
LTR was observed with 10 continuous repetitions (figure 6). The participants did not receive any other treatment modalities at the time of the DDN.

\section{STATISTICAL ANALYSIS}

SPSS version 16 was utilized for statistical analysis of the data. The one-sample Kolmogorov-Smirnov test was conducted to assess the normal distribution of all the independent variables. Paired t-test was used for detecting any significant changes between the pre and post treatment of all variables in the present study. The confidence level of statistical significance was determined at $\alpha \leq .05$.

\section{RESULTS}

13 subjects participated in the present study. All variables demonstrated a normal distribution upon analysis with the One-sample Kolmogorove-Smirnov test. The anthropometric features of the participants are shown in Table I. Results from the pre-and post-intervention analysis are demonstrated in Table II.

Notably, the most clinical and ultrasonographic outcome measures were significantly $(\mathrm{P}<.05)$ changed after the treatment in the present study. Decrease of intensity in pain was

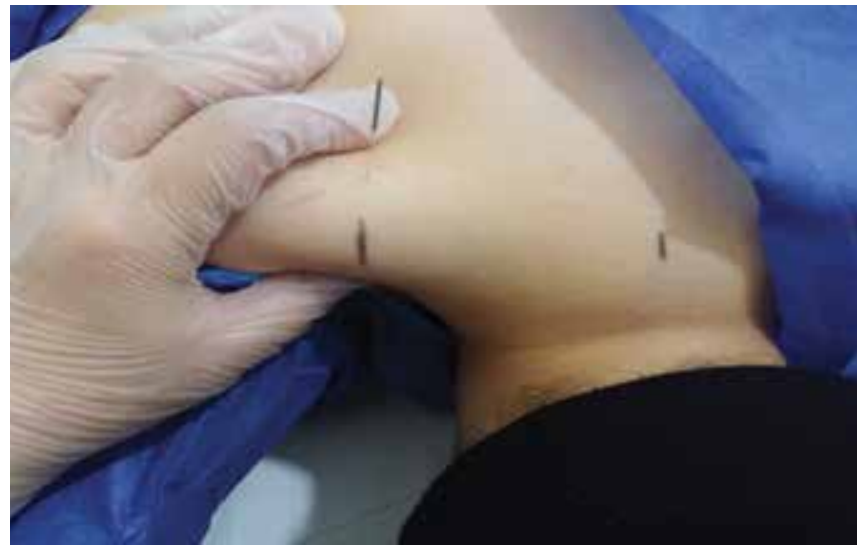

Figure 6. DDN of active trigger point in the upper trapezius muscle.

observed after treatment in participants $(\mathrm{P}<.001)$. There was a significant improvement in PPT after the intervention $(\mathrm{P}<.001)$. Also the baseline and after intervention values for contralateral side bending $\mathrm{ROM}$ showed a significant improvement for this variable $(\mathrm{P}<.008)$. The range of ipsilateral side bending improved but the differences were not significant $(\mathrm{P}=.73)$. There was significant improvement $(\mathrm{P}<.001)$ in the intramuscular blood circulation after inter-

Table I. Anthropometric feature of participants (N: 13).

\begin{tabular}{lllll}
\hline & Age $($ year $)$ & Height $(\mathbf{c m})$ & Weight $(\mathbf{k g})$ & BMI $(\mathbf{k g} / \mathbf{m} 2)$ \\
\hline Mean \pm SD & $28.85 \pm 7.70$ & $163.69 \pm 6.07$ & $60.07 \pm 11.67$ & $22.32 \pm 3.58$ \\
$($ Range $)$ & $(20-44)$ & $(150-171)$ & $(46-85)$ & $(18.06-29.02)$ \\
\hline
\end{tabular}

BMI= Body Mass Index; SD= Standard deviation

Table II. The results of Paired t- test between pre and post conditions in the present study $(\mathrm{N}=13)$.

\begin{tabular}{llll}
\hline Variable & Baseline & Post treatment & P value \\
\hline VAS & $8.23 \pm 0.72$ & $5.69 \pm 0.85$ & $.000^{*}$ \\
\hline PPT & $7.83 \pm 2.8$ & $13.22 \pm 3.2$ & $.000^{*}$ \\
\hline Ipsilateral ROM & $35.30 \pm 5.42$ & $36.84 \pm 5.56$ & .73 \\
\hline Contra lateral ROM & $32.23 \pm 3.58$ & $38.23 \pm 4.54$ & $.008^{*}$ \\
\hline Thickness at rest & $11.65 \pm 1.9$ & $13.02 \pm 2.5$ & .26 \\
\hline Area of TP & $20.76 \pm 6.13$ & $21.92 \pm 4.76$ & .42 \\
\hline PSV & $11.74 \pm 2.03$ & $7.59 \pm 2.18$ & $.000^{*}$ \\
\hline EDV & $1.7 \pm 0.49$ & $2.86 \pm 0.58$ & $.000^{*}$ \\
\hline RI & $0.84 \pm 0.51$ & $0.59 \pm 0.14$ & $.000^{*}$ \\
\hline Stiffness (strain ratio) & $11.53 \pm 1.60$ & $14.00 \pm 1.52$ & $.000^{*}$ \\
\hline A
\end{tabular}

Abbreviations: SEM: standard error of measurement, PSV: peak systolic velocity, EDV: End diastolic velocity, RI: Resistive Index. Significant p value was shown by *. 
vention. There was not only a significant decrease in PSV and $\mathrm{RI}(\mathrm{P}<.001)$ but also an increase in EDV $(\mathrm{P}<.001)$. Moreover, in muscle stiffness at the rest state, analysis showed a significant reduction after intervention $(\mathrm{P}<.05)$. The upper trapezius muscle thickness $(\mathrm{P}=.26)$ and the area of $\mathrm{MTr} P$ were not significantly different after DDN $(\mathrm{P}=.42)$.

\section{DISCUSSION}

Present study showed a decrease in the upper trapezius muscle stiffness and an increase in blood flow due to DDN intervention in women with MPS. These findings of the current study provide objective evidence of the effectiveness of DDN. Also, the improvement in clinical parameters (including decrease of VAS, increase of PPT and side bending cervical $\mathrm{ROM}$ ) were consistent with ultrasonographic parameters, indicating that the DDN have immediate therapeutics effects on the MTrP.

The present study may be the first comprehensive (objective and subjective) assessment of the effects of DDN in upper trapezius affected with active MTrP. The upper trapezius ultrasonographic scanning findings were seen post treatment of DDN, suggesting the possibility of clinical outcomes confirmation and quantification of treatment effects using ultrasonography. A major limitation of the present study is the lack of an untreated patient control group, thus changes in clinical findings could have been due to placebo effect or other factors not related to the DDN. In the present study, only the immediate effects of DDN on female subjects were examined and lack of long term intervention and follow up should be acknowledged.

There is a common agreement that over-use or sustained muscle contractions may lead to changes in the intramuscular blood circulation and formation of MTrP $(1,12)$. According to the normal physiological mechanism, intramuscular circulation is dependent on muscle contraction or relaxation. Blood flow is enhanced by rhythmic muscle contractions with muscular pump and is diminished by sustained muscle contraction which compresses the vessels and increase peripheral resistance (1). Based on the "energy crisis theory" that was proposed by Simons, the formation of a trigger point is related to an excessive release of acetylcholine and calcium (3). These substances induce continuous muscle contraction and an ischemic condition around the trigger points (9). Present study demonstrated an increase in blood flow due to DDN intervention in patients with MPS. A significant reduction of RI after needling could confirm that this method has immediate effects on blood flow. Recently, Cognee et al conducted a study in healthy participants and shown an increase in upper trapezius blood flow due to dry needling (13). Also, in their study the dura- tion of influence of DDN on the upper trapezius blood flow was examined and they confirmed that dry needling could significantly increase blood flow at the insertion point up to 15 min after needling (13). Concerning different measurement methods with the mentioned research the present study findings in response to DDN are in accordance with them. One of the probable mechanisms that explains the changes in blood flow in response to DDN is related to activation of $c$-fibers and A- $\delta$. This axon reflex activates the release of some substances that lead to vasodilatation in vessels and increase in muscle blood flow $(9,13)$. Temporary increase in sympathetic nerve activity via sympathetic stress response was presented as a result of an increase in blood flow in the contralateral upper trapezius, in response to needling in Sandberg et al (8).

In current study, findings demonstrated a significant reduction of the upper trapezius stiffness after DDN. Although previous studies have revealed that after dry needling, palpable stiffness was reduced, quantification of this parameter after treatment has been done in only a few studies. Utilizing a dissimilar elasticity imaging technique associated with ultrasound, Maher et al. (12) examined the effect of dry needling on the upper trapezius using shear-wave elastography in females with MPS and reported a significantly reduced stiffness in this group; the present findings are in agreement with them. Shear wave elastography may be generally more accepted than ultrasound elastogram. Unfortunately, our device had only the ultrasound elastogram software for measuring muscle hardness. Measuring muscle stiffness by shear wave elastography is recommended for future studies. In another study, the stiffness of the upper trapezius was evaluated after acupuncture and electro-acupuncture and non-significant reduction after treatment in both interventions were reported (2). Those authors' examination was done with similar elasticity imaging technique using ultrasound elastography. It seems that stimulation of needle and production of LTR in DDN have mechanical effects on the MTrP. Indeed, LTR causes mechanical disruption of MTrP, localized stretch to the contractured cytoskeletal structures and alteration in the muscle fiber tension and length $(9,13)$. According to the analysis of gray scale images, non-significant increase in the area of trigger point and upper trapezius thickness at rest condition was seen (figure 7). Only one study was found in this field and the present study's findings are dissimilar to the ones that reported a decreased area of trigger points after treatment by acupuncture and electro acupuncture in the upper trapezius (2). It is likely that, increases in the blood flow that was seen in the current research and the presence of inflammatory substances after needle stimulation of this point, may lead to edema and larger size. Non-significant increase in the upper trapezius thick- 


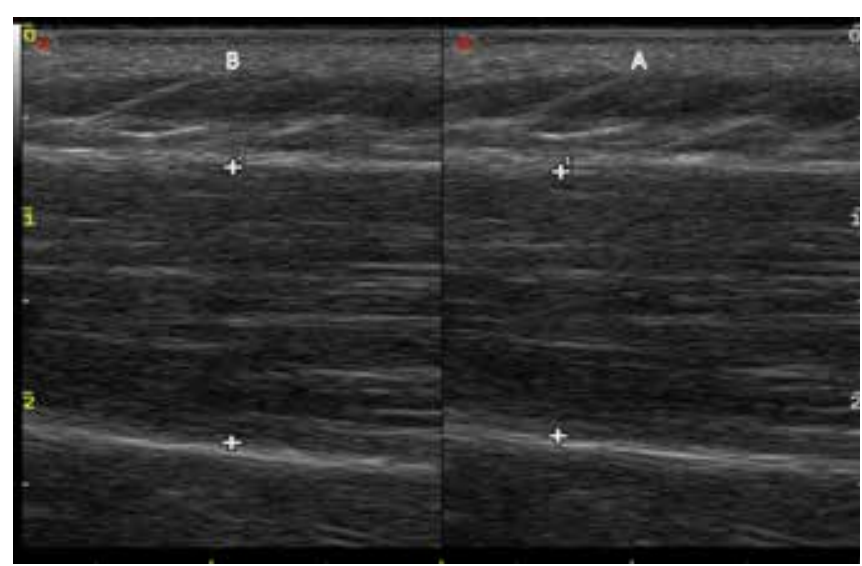

Figure 7. B-mode images from the same volunteer A) before and B) after treatment with DDN. Increased upper trapezius thickness after treatment is shown. Muscle thickness were marked in the images.

ness at resting condition was reported and no published research was found about this issue. It seems that increase in the thickness of the upper trapezius after DDN, is related to increased blood flow and edema. More studies are needed in this area to assess the correlation of blood flow parameters, trigger point size, and muscle thickness after several sessions of needling.

According to the results presented, current study showed clinical effects of DDN in treatment of patients with MPS on perception of pain. In current study, pain is the main clinical outcome and for measuring of it, VAS was used. VAS is one of the methods that is easy to use, and valid, and also used in most researches by clinicians (15). After intervention, subjective pain intensity was decreased. The assessment of pain intensity and PPT are common measures that were examined after DDN in many studies. The findings of the present study are similar to that of other studies which reported a reduction in pain intensity, increase in PPT and bilateral cervical side bending ROM as immediate effects of DDN $(6,15)$. Actually, the spontaneous pain and hyper sensitivity of active MTrPs are one aspect of their sensory abnormalities. The presence of some biochemical substances in the active MTrP environment was confirmed by Shah et al (10). These substances are associated with tenderness and persistent pain. The stimulation of LTR by needling may alter these substances, deactivate nociceptors or activate the descending inhibitory pain system. These mechanisms could have an effect on the pain intensity, PPT, and ultimately deactivate MTrP $(9-10,13)$. On the other hand, the minimal significant clinical difference for pain intensity on VAS is $12 \mathrm{~mm}$ (6). In the present study, $25 \mathrm{~mm}$ reduction was reported on VAS. According to the results, PPT increased after DDN. An increase of about $20-25 \%$ is ideal for this parameter (15). The current study showed a $45 \%$ increase. These changes indicate that the DDN approach had a clinical effect on the intensity and threshold of pain of the upper trapezius which is involved in MPS.

\section{CONCLUSION}

This study showed that DDN had immediate influences on clinical symptoms and upper trapezius ultrasonographic features in females with MPS. Evaluation of influences of this technique on other muscles is recommended and Larger samples are needed to clarify the relevant pathophysiological mechanism of interventions. The effects of individual anthropometric features such as gender, body mass index (BMI) and age on muscle stiffness, circulation, and morphological properties should be taken into consideration in future studies. Among the parameters of Doppler ultrasound, the reliability of measuring EDV (ICC) is not excellent. So, the evaluation and extension of accurate Doppler ultrasonographic parameters to measure blood flow is recommended for future studies.

\section{CONFLICTS OF INTEREST}

The authors declare no conflicts of interest.

\section{ACKNOWLEDGEMENTS}

This study was conducted at the Federation of Sports Medicine, and the authors appreciate the officials of this center for their facilities and cooperation.

\section{FUNDING}

This study was supported by Tehran University of Medical Sciences and Health services grant 94-02-32-29449.

\section{ABBREVIATIONS}

MPS: Myofascial pain syndrome; MTrP: myofascial trigger points; DDN: deep dry needling; ROM: range of motion; LTR: local twitch response; ROI: regions of interests; PSV: Peak Systolic Velocity; EDV: End Diastolic Velocity; RI: Resistive Index; PPT: pain pressure threshold

\section{REFERENCES}

1. Ballyns JJ, Shah JP, Hammond J, Gebreab T, Gerber LH, Sikdar S. Objective sonographic measures for characterizing myofascial trigger points associated with cervical pain. J Ultrasound Med. 2011;30:1331-1340. 
2. Müller CEE, Aranha MFM, Gavião MBD. Two-Dimensional Ultrasound and Ultrasound Elastography Imaging of Trigger Points in Women with Myofascial Pain Syndrome Treated by Acupuncture and Electroacupuncture: A Double-Blinde Randomized Controlled Pilot Study. Ultrason imaging. 2014;0161734614546571.

3. Simons D, Travell J. Simons' myofascial pain and dysfunction: the trigger point manual, vol. 2. In: Baltimore. Lippincott Williams \& Wilkins.1999.

4. Sikdar S, Shah JP, Gebreab T, Yen R-H, Gilliams E, Danoff J, et al. Novel applications of ultrasound technology to visualize and characterize myofascial trigger points and surrounding soft tissue. Arch Phys Med Rehabil. 2009;90:1829-1838.

5. Fernández-de-las-Peñas C, Dommerholt J. Myofascial trigger points: peripheral or central phenomenon? Curr Rheumatol Rep. 2014;16:1-6.

6. Ong J, Claydon LS. The effect of dry needling for myofascial trigger points in the neck and shoulders: A systematic review and meta-analysis. J Bodyw Mov Ther. 2014;18:390-398.

7. Shah J, Heimur J. New frontiers in the pathophysiology of myofascial pain. Pain. 2012;22:27.

8. Sandberg M, Larsson B, Lindberg LG, Gerdle B. Different patterns of blood flow response in the trapezius muscle following needle stimulation (acupuncture) between healthy subjects and patients with fibromyalgia and work-related trapezius myalgia. Eur J Pain. 2005;9:497-497.

9. Shah JP, Gilliams EA. Uncovering the biochemical milieu of myofascial trigger points using in vivo microdialysis: an application of muscle pain concepts to myofascial pain syndrome. J Bodyw Mov Ther. 2008;12:371-384.

10. Shah JP, Danoff JV, Desai MJ, Parikh S, Nakamura LY, Phillips TM, et al. Biochemicals associated with pain and inflammation are elevated in sites near to and remote from active myofascial trigger points. Arch Phys Med Rehabil. 2008;89:16-23.

11. Gerber LH, Sikdar S, Armstrong K, Diao G, Heimur J, Kopecky J, et al. A systematic comparison between subjects with no pain and pain associated with active myofascial trigger points. PM\&R 2013;5:931-938.

12. Maher RM, Hayes DM, Shinohara M. Quantification of dry needling and posture effects on myofascial trigger points using ultrasound shear-wave elastography. Arch Phys Med Rehabil. 2013;94:2146-2150.

13. Cagnie B, Barbe T, De Ridder E, Van Oosterwijck J, Cools A, Danneels L. The influence of dry needling of the trapezius muscle on muscle blood flow and oxygenation. J Manipulative Physiol Ther. 2012;35:685-691.
14. Fleckenstein J, Zaps D, Rüger LJ, Lehmeyer L, Freiberg F, Lang PM, et al. Discrepancy between prevalence and perceived effectiveness of treatment methods in myofascial pain syndrome: results of a cross-sectional, nationwide survey. BMC Musculoskelet Disord. 2010;11:32.

15. Kietrys DM, Palombaro KM, Azzaretto E, Hubler R, Schaller B, Schlussel JM, et al. Effectiveness of dry needling for upper-quarter myofascial pain: a systematic review and meta-analysis. J Orthop Sports Phys Ther. 2013;43:620-634.

16. Cummings TM, White AR. Needling therapies in the management of myofascial trigger point pain: a systematic review. Arch Phys Med Rehabil. 2001;82:986-992.

17. Gerber LH, Sikdar S, Aredo JV, Armstrong K, Rosenberger WF, Shao H, et al. Beneficial Effects of Dry Needling for Treatment of Chronic Myofascial Pain Persist for 6 Weeks After Treatment Completion. PM\&R 2016.

18. Myburgh C, Larsen AH, Hartvigsen J. A systematic, critical review of manual palpation for identifying myofascial trigger points: evidence and clinical significance. Arch Phys Med Rehabil. 2008;89:1169-1176.

19. Basford JR, An K-N. New techniques for the quantification of fibromyalgia and myofascial pain. Curr Pain Headache Rep. 2009;13:376-378.

20. Brandenburg JE, Eby SF, Song P, Zhao H, Brault JS, Chen $\mathrm{S}$, et al. Ultrasound elastography: the new frontier in direct measurement of muscle stiffness. Arch Phys Med Rehabil. 2014;95:2207-2219.

21. Padulo J, Oliva F, Frizziero A, Maffulli N. Muscle, Ligaments and Tendons Journal-Basic principles and recommendations in clinical and field science research: 2016 update. Muscles Ligaments Tendons J. 2016;6(1):1-5.

22. Fryer G, Hodgson L. The effect of manual pressure release on myofascial trigger points in the upper trapezius muscle. J Bodyw Mov Ther. 2005;9:248-255.

23. Niitsu M, Michizaki A, Endo A, Takei H, Yanagisawa O. Muscle hardness measurement by using ultrasound elastography: a feasibility study. Acta radiol. 201;52:99-105.

24. Adigozali H, Shadmehr A, Ebrahimi E, Rezasoltani A, Naderi F. Reliability of assessment of upper trapezius morphology, its mechanical properties and blood flow in female patients with myofascial pain syndrome using ultrasonography. J Bodyw Mov Ther. 2017;21:35-40

25. Adigozali H, Shadmehr A, Ebrahimi E, Rezasoltani A, Naderi F. Ultrasonography for the assessment of the upper trapezius properties in healthy females: a reliability study. Muscles Ligaments Tendons J. 2016;6:167. 